\title{
Does the Nucleoid Determine Cell Dimensions in Escherichia coli?
}

\author{
Arieh Zaritsky ${ }^{*}$, Waldemar Vollmer ${ }^{2}$, Jaan Männik ${ }^{3}$ and Chenli Liu ${ }^{4}$ \\ ${ }^{1}$ Faculty of Natural Sciences, Ben-Gurion University of the Negev, Beersheba, Israel, ${ }^{2}$ Centre for Bacterial Cell Biology, \\ Institute for Cell and Molecular Biosciences, Newcastle University, Newcastle upon Tyne, United Kingdom, ${ }^{3}$ Department \\ of Physics \& Astronomy, The University of Tennessee, Knoxville, Knoxville, TN, United States, ${ }^{4}$ Shenzhen Institute of \\ Synthetic Biology, Shenzhen Institutes of Advanced Technology, Chinese Academy of Sciences, Shenzhen, China
}

OPEN ACCESS

Edited by:

Kenneth C. Keiler,

Pennsylvania State University,

United States

Reviewed by:

Randy Morgenstein,

Oklahoma State University,

United States

Daniel Haeusser,

Canisius College, United States

*Correspondence.

Arieh Zaritsky

ariehzar@gmail.com

Specialty section:

This article was submitted to Microbial Physiology and Metabolism,

a section of the journal

Frontiers in Microbiology

Received: 17 April 2019

Accepted: 11 July 2019

Published: 06 August 2019

Citation:

Zaritsky A, Vollmer W, Männik J and Liu C (2019) Does the Nucleoid

Determine Cell Dimensions

in Escherichia coli?

Front. Microbiol. 10:1717.

doi: 10.3389/fmicb.2019.01717
Bacillary, Gram-negative bacteria grow by elongation with no discernible change in width, but during faster growth in richer media the cells are also wider. The mechanism regulating the change in cell width $W$ during transitions from slow to fast growth is a fundamental, unanswered question in molecular biology. The value of $W$ that changes in the divisome and during the division process only, is related to the nucleoid complexity, determined by the rates of growth and of chromosome replication; the former is manipulated by nutritional conditions and the latter-by thymine limitation of thyA mutants. Such spatio-temporal regulation is supported by existence of a minimal possible distance between successive replisomes, so-called eclipse that limits the number of replisomes to a maximum. Breaching this limit by slowing replication in fast growing cells results in maximal nucleoid complexity that is associated with maximum cell width, supporting the notion of Nucleoid-to-Divisome signal transmission. Physical signal(s) may be delivered from the nucleoid to assemble the divisome and to fix the value of $W$ in the nascent cell pole.

Keywords: cell cycle and dimensions, nucleoid structure and complexity, nutritional shifts, transertion, eclipse, physical effector

\section{INTRODUCTION}

Rod-shaped bacteria such as Escherichia coli grow by elongation without a discernible change in width (Trueba and Woldringh, 1980; Taheri-Araghi et al., 2014), but when growing faster in richer media (at a constant temperature) the cells are both, longer and wider (Schaechter et al., 1958; Zaritsky, 1975a). The nature of the mechanism that regulates the change in cell width during a transition from slow to fast growth, so-called nutritional shift-up, is a fundamental but still unanswered question in bacteriology (Zaritsky and Woldringh, 2015). In this brief Perspective, putative mechanisms to control cell width during shift-up and other transitions are discussed, with perspective for future research to test the predictions emanating from new, bold ideas.

Most mutants affecting cell dimensions involve the biosynthetic pathway of peptidoglycan (PG) - the shape-maintaining macromolecule and the bacterial cytoskeleton, including the actinlike MreB and the tubulin-like FtsZ, and their associated cell morphogenesis proteins (Typas et al., 2012). MreB is essential for cell elongation. Filaments of MreB rotate around the short axis of the cell, driven by peptidoglycan synthesis (van Teeffelen et al., 2011), and certain mutations in MreB 
cause irregular cell shape and/or altered, most often increased cell diameter (Shi et al., 2018; Kurita et al., 2019). By contrast, FtsZ is essential for cell division. It moves around the cell division plane by treadmilling to organize the divisome complex that synthesizes the new cell poles during cell division (Bisson-Filho et al., 2017; Yang et al., 2017).

In this Perspectives article we hypothesize that cytoskeleton dynamics and PG biosynthesis function to maintain cell dimensions in response to a yet elusive, primary and perhaps mechanical signal to determine cell width, a process that starts during physiological transitions at the new cell pole. Some compelling evidence indicates that such an early signal is related to the Bacterial Cell Division Cycle, so-called the BCD dogma (Helmstetter et al., 1968). A brief description of this dogma is provided below.

\section{THE BACTERIAL CELL DIVISION CYCLE}

Prokaryotic chain-synthesis rates of the three macromolecules involved in the flow of genetic information are constant, at least at $37^{\circ} \mathrm{C}$, independent of the total mass growth rate. Transcribed mRNA is simultaneously co-translated with its gene sequence at modulus 3-matched rates, roughly 17 amino acids and 50 base pairs per second, respectively, by polysomes (Maaløe and Kjeldgaard, 1966). Another essential hyper-structure (Norris et al., 2007), the replisome replicates the $4.6 \mathrm{Mbp}$ circular chromosome bidirectionally from oriC to terC as fast (O’Donnell et al., 2013) as 2,000 bp sec ${ }^{-1}$, over 10-fold faster than mammalian DNA replication fork, and with an amazingly low frequency of $10^{-8}$ mistakes. The times taken to complete (a) a round of replication $C$ and (b) the subsequent cell division $D$ are roughly constant, about 40 and $20 \mathrm{~min}$, respectively, regardless of the total mass doubling time $\tau_{m}$ (under $60 \mathrm{~min}$ at $37^{\circ} \mathrm{C}$ ) (Helmstetter et al., 1968; Jiménez-Sánchez, 2018). Replication round is initiated when cell mass reaches a value $M i$ per oriC number (Donachie, 1968; Pritchard et al., 1969), at the mother cell (i.e., cell cycles overlap) when $\tau_{m}<(C+D)$. At faster growth rates, when $\tau_{m}<C$, a new replication cycle inaugurates at ori $C$ before the previous one ends at ter $C$ (i.e., replication cycles overlap), thus forming multi-forked replicating chromosomes, sometimes (when $\tau_{m}<\sim 20 \mathrm{~min}$ ) (Taheri-Araghi et al., 2014) even at the grandmother's cycle. (See Appendix for definitions of parameters and field-specific terms.)

\section{BACTERIAL DIMENSIONS AND THE CELL DIVISION CYCLE}

Faster growing cells are larger because they divide a constant time $[(C+D)=\sim 60 \mathrm{~min}]$ after initiating the chromosome replication (Helmstetter et al., 1968) at a constant mass Mi (Donachie, 1968; Pritchard et al., 1969; Amir, 2014). Replication is linear whereas mass [or volume, since density is constant (Kubitschek et al., 1984)] is synthesized exponentially at a rate inversely proportional to $\tau_{m}$ (Koch, 1993). The dissociation between rates of growth and of replication was confirmed by extending $C$ (slowing replication) by limiting the concentration of thymine [T] supplied in the growth medium of thymine-requiring mutants (Pritchard and Zaritsky, 1970). It was reassuring to find that this so-called thymine limitation results in larger average cell mass (Zaritsky and Pritchard, 1973) consistent with $M=\ln 2 \times M i \times 2^{(C+D) / \tau}$. This increased size of cells growing at an identical rate was anticipated $a$ priori to be manifested by longer cells because they usually grow by elongation only; it was highly surprising to find that such thymine-limited cells are wider as well, just as faster growing cells are (Zaritsky and Pritchard, 1973). The common denominator to the two conditions at which cells are wider, shorter $\tau_{m}$ at a constant $C$ and longer $C$ at a constant $\tau_{m}$, is the number of replication positions (Sueoka and Yoshikawa, 1965) $n=C / \tau$. This parameter was used to define nucleoid complexity NC (Zaritsky et al., 2006) the culture-average amount of DNA in genome equivalents associated with a single terC (Woldringh et al., 1990) G/terC\# $=(\tau / C \times \ln 2)\left(2^{C / \tau}-\right.$ $1)=\left(2^{n}-1\right) /(n \times \ln 2)$; larger $N C$ implies a larger nucleoid. The satisfactory correlation observed between cell width $W$ and NC (Zaritsky, 2015; Campos et al., 2018) led to the idea (Zaritsky and Woldringh, 2015; Zaritsky et al., 2017) that cell length $L$ is passively determined by the exponential rate of mass synthesis ( = volume growth) and active regulation of cell width by a putative signal that is transmitted from the nucleoid to the PG-synthetic machinery.

\section{DIVISION RATE AND WIDTH: CHANGES DURING NUTRITIONAL SHIFT-UP}

The classical experiment of nutritional shift-up to faster growth (Kjeldgaard et al., 1958) discovered temporary and orderly dissociations between the main synthetic activities, the most striking of which is the so-called "rate maintenance" of cell division that keeps at the pre-shift speed for about $65 \mathrm{~min}$. This phenomenon was readily explained by the results that led to the BCD dogma (Helmstetter et al., 1968), and clarified the dependence of $M$ and $G$ on $\tau_{m}$ under constant $M i, C$ and $D$ (Donachie, 1968; Pritchard et al., 1969). Studying cell dimensions during a similar transition (Woldringh et al., 1980) likewise disclosed that the change in cell width occurs exclusively during the division process and at the division site: $L$ continues to extend at the pre-shift rate until the first division, during which process (and only then) cell width $W$ rises as well, locally at the divisome. This local change results in temporary pear-shaped, tapered cells (Zaritsky and Woldringh, 2015; Zaritsky et al., 2017). The new $W$ equalizes along $L$ and during the following growth and divisions thus recovering their straight cylindrical shape with new dimensions that fit the post-shift $\tau_{m}$; the equalizing process is slow likely because the adjustment in the widths of the net-like sacculus requires growth of the cell (Höltje, 1998).

Thus, two types of PG-synthetic activities must exist in separate hyper-structures (Norris et al., 2007): elongasome, operating along the cylindrical PG during L-extension, that keeps cell width constant, and divisome that operates perpendicularly during cell division and allows changes in $W$ (Van der Ploeg et al., 2003). 


\section{TEMPORAL AND SPATIAL ASPECTS OF THE DIVISOME AND ELONGASOME ACTIVITIES}

Normal cell division is regulated by the nucloid, both temporally and spatially (Zaritsky and Woldringh, 2015; Zaritsky et al., 2017): it happens after chromosome replication is terminated and precisely between the two segregating nucleoids. Similarly, cell width $W$ seems to be determined by the nucleoid in both arenas: it rises during the division process - and at the divisome only.

The mechanism that blocks cell division before two equal sets of its full genetic information are available by completing replication is under intense investigations (Männik and Bailey, 2015), but the one fixing $W$ is neglected, likely due to the enormous variation among species, even strains within $E$. coli (Begg and Donachie, 1978). Regulation of cell width is likely complex due to the large number of downstream processes leading from the presumed primary signal(s) to the executing PG synthesizing machinery (Egan et al., 2017). Many mutants in the genes coding for the involved proteins would therefore change these pathways and hence, cell width or shape. For example, certain mutants with changes in $\mathrm{MreB}$, which result in an altered helical pitch angle of the MreB cytoskeleton, have larger cell widths (Ouzounov et al., 2016). It was not reported if these cells have changed their growth rate or NC. It is also not known if cells with poorly functioning MreB or cells grown with sublethal concentrations of the MreB inhibitor A22 (Ouzounov et al., 2016) correlate their (abnormal) cell width with $N C$ within a range of growth rates, as do cells with unaltered MreB. The widths of B. subtilis cells can be altered by increasing the level of certain PG synthases, which does not change the growth rate of the cells (Dion et al., 2019). Again, it is not known if cells disturbed in this way maintain the correlation between $N C$ and cell width at different growth rates.

\section{NATURE OF THE PRIMARY SIGNAL -NEED FOR A NEW PARADIGM}

Various ideas have been entertained as signals to initiate the biochemical cascade of reactions leading to activation of the divisome, all in the realm of regulatory molecules (Egan and Vollmer, 2013; Du and Lutkenhaus, 2017). A physical element is preferred (Rabinovitch et al., 2003; Zaritsky and Woldringh, 2015), by analogy to the mode of thinking that brought about the "enzyme-cannot-make-enzyme (e-c-m-e) paradox" (Stent, 1968; Stent and Calendar, 1978): based on the knowledge in the 1930s, the omni-potent, highly variable proteins were seriously considered as the store of genetic information whereas the monotonous structure of DNA led the scientists to think of it as a mere reservoir of nucleotides. The theoretical "e-c-m-e paradox," together with convincing discoveries of DNA structure and function resulted in The Central Dogma of Molecular Biology and understanding the flow of genetic information unidirectionally from nucleic acids to proteins (Stent, 1968). The structure of prokaryotic DNA as the bacterial nucleoid and convincing physiological studies (Helmstetter et al., 1968) exposed coupling between its replication / segregation with duplication of the other unique cellular macromolecule / structure, the PG sacculus, by cell division. The yet-to-bedisclosed mechanism that governs this coupling seems to need a new concept, one that is external to the never-ending search (e.g., Männik et al., 2018) for the primary signal by a "regulator-ofthe-regulator paradox." In other words: what is the nature of the division regulator that is at the top of the hierarchy? The template feature came from another discipline (information science) than chemistry (producing an enzyme); by analogy, triggering cell division may stem from physics-or another discipline that we are not aware of currently rather than the proteins involved in the division process itself. Can the divisome activation be triggered by the nucleoid's complexity or replication status?

Two articles (Knox and Funk, 2014; Knox, 2018) introduced biophysical signaling "as having a central role in cancer through influences on cell proliferation, cell cycle progression, apoptosis, cell migration and orientation, as well as cell differentiation." Moreover, "Many aspects of the cell cycle and systemic functioning are regulated by biophysical (bioelectric) signals. These include cell division and proliferation, embryonic development (e.g., left-right body asymmetry, axon outgrowth), epithelial wound healing, tissue regeneration and cancer cell migration." It is proposed here that in bacteria too biophysical cues take major roles in signaling basic functions such as cell division and width determination at the divisome.

A hypothetical physical signal, transmitted from the nucleoid to the divisome that simultaneously activates cell division and determines cell width has been invoked as primary (Rabinovitch et al., 2003), involves the so-called "transertion" process (Woldringh, 2002): co-transcriptional translation of membrane protein genes coupled to insertion of these proteins to the membrane. The envelope is thus pulled toward the nucleoid and stressesed along cell length in a direction that changes during the last stage of the replication cycle. This change is presumed to be sensed by the cell to trigger the assembly of the divisome (Rabinovitch et al., 2003).

\section{THYMINE- LIMITATION AND -STEP TRANSITIONS}

Immediate reaction to [T]-step-down in thymine concentration slows replication (Pritchard and Zaritsky, 1970) (extends C) hence reduces division frequency due to postponed terminations of ongoing replication cycles. Continued exponential mass growth at identical rate of divisome-delayed cells results in larger $M$ and temporarily longer cells (Zaritsky and Pritchard, 1973), just as nutritionally up-shifted cells overshoot their new steady-state $L$ (Kjeldgaard et al., 1958). In both cases, the default mass growth is accommodated by the continued function of the elongasome, and $W$ starts to rise later (Zaritsky et al., 2017), when the deferred divisomes are assembled following the delayed terminations. At faster growth of $\mathrm{Thy}^{+}$strains, the longer time to complete the larger division septum is compensated by the faster rate of septum build-up, culminating by a manifested constant $D$ period (Zaritsky et al., 1999). In contrast, compensation does not 
exist at identical growth rate in cells that are wider due to slow replication, and hence $D$ is longer. A new steady-state is predicted to arise, with bigger cells due to the extended $D$. This scenario indeed happens under slower growth, in glycerol-supplemented minimal salts medium $\left(\tau_{m}>\sim 60 \mathrm{~min}\right)$.

This "simple" scenario, however, does not occur in glucosesupplemented, relatively fast-growing $\left(\tau_{m}<\sim 50 \mathrm{~min}\right)$ thy $A$ mutants under thymine limitation (at low [T]) (Zaritsky and Pritchard, 1973). Under such circumstances, cells do not reach steady-state dimensions: the mean inter-division time $\tau_{d}$ is longer than the constant $\tau_{m}$, hence mean culture cell size increases at a rate $\mathrm{d} M / \mathrm{d} \tau$ that is proportional to $\left(\tau_{d}-\tau_{m}\right)$, which in turn depends inversely on the value of $C$ that is [T]-dependent. Qualitatively, this phenomenon was explained by a sort of vicious circle (Zaritsky et al., 2017): extended $D$ due to wider cells results in larger cells, that in turn further extends $D$, but this explanation completely ignores the question posed here: what causes the divisome in the first place to build a wider circumference when the number of replication positions $n=C / \tau$ is larger.

The slow rise in cell width $W$ under these circumstances is, however, limited to a maximum, at which the increased cell size is accommodated by branching as well as extended $L$ (see e.g., Figure 2 in Zaritsky et al., 2007). Putative link between $N C$ and $W$ may resolve this fundamental question; it predicts a maximum achievable width because of so-called eclipse effect, as described below.

\section{THE ECLIPSE CONCEPT}

The unusual large and monstrous-shape of cells that slowly evolve during long periods of growth under this non-steady state conditions is reversible, and very fast, too: restoring replication rate using the thymine step-up regime temporarily but markedly enhance the frequency of divisions (Zaritsky et al., 2011); while $\tau_{m}$ remains constant, $\sim 40 \mathrm{~min}$ at all [T] (above a certain strain- and medium -dependent threshold) (Zaritsky and Pritchard, 1971, 1973), divisions proceed almost synchronously at $\sim 20 \mathrm{~min}$ intervals for several cycles (at least 5 under the recorded circumstances). This is consistent with existence of another phenomenon: a minimal physical distance $l_{\text {min }}$ along the chromosome length $\Lambda$ needed for a replisome to be away from oriC before a new replisome can initiate a subsequent replication round there (Zaritsky et al., 2007). In units of time, this so-called eclipse period $E$ is proportional directly to the fraction $l_{\min } / \Lambda$ and to $C$, the time taken to replicate the whole $\Lambda$, i.e., $E=C\left(l_{\min } / \Lambda\right)$. This idea was originally conceived by observing results of totally different nature (Zaritsky, 1975b), substantiated and coined as eclipse a decade ago (Zaritsky et al., 2007), and experimentally confirmed recently by others (Khan et al., 2016).

To sum up, there are two seemingly contradictory observations: (a) rise, albeit slow, in cell width $W$ during thymine limitation of fast growing thy $A$ strains and (b) a limit to $W$ that cause cells to elongate and branch when breached. These two are qualitatively consistent with existence of, respectively, (a) regulation of cell width by nucleoid complexity NC (symbolized as $N C \rightarrow W$ ) that is affected at the divisome during the division process only, and (b) a maximum value for $N C$ that is limited by the eclipse $E$. The latter is manifested under slow replication rate at low [T] 's, when $\tau_{m}<<C$, (e.g., $2 \tau_{m}<C$ ), conditions that set a maximum number of simultaneously acting replisomes on a nucleoid resulting in $E=\left(\tau_{d}-\tau_{m}\right)>0$. Thus, if indeed $N C \rightarrow W, E$ serves as a tool to manipulate (a) and explain (b), at least partially and qualitatively.

The $N C \rightarrow W$ hypothesis gains support from a recent article (Campos et al., 2018), suggesting "that the size of the nucleoid is an important element of the coordination mechanism between cell morphogenesis and the cell cycle." The comprehensive data included may contain mutants which deviate from the model and so would point to proteins that are involved in the downstream processes of the mechanism involved.

\section{CAN GENE DOSAGE EXPLAIN THE $N C \rightarrow W$ HYPOTHESIS?}

$N C$ is also reflected by gene dosage. Two quantities can be distinguished in a steady state cell population: gene concentration is the number of genes per cell mass (or volume) $2^{-n x} / M i$, where $x$ is the distance of a gene from oriC, and relative gene dosage, defined as the number of gene copies per total genome content $2^{n(1-x)} / N C$ (Chandler and Pritchard, 1975). Both quantities depend exponentially on the gene location on the chromosome. Concentrations of all genes except oriC decrease as $N C$ rises. The decrease depends on the position of the said gene on the genetic map; it drops as the gene is further downstream oriC and is largest for ter $C$. The numbers of oriC-proximal genes are enriched compared to those of terC-proximal genes as $\mathrm{NC}$ rises. It was recently argued that relative gene dosage is the relevant parameter that determines the gene's expression level (Lin and Amir, 2018). This argument stems from the assumption that the protein-synthesizing-system is rate-limiting for gene expression in E. coli. This assumption has, however, not been thoroughly tested yet. Thymine-limited cells could easily be used to test and advance these ideas further.

Variation of gene dosage across genome at different growth conditions can lead to changes in expression profiles of genes involved in cell wall synthesis, many of which are scattered, including oriC-proximal regions. Composition and cross-linking of septal cell wall can be expected to be affected by differential production levels of such cell wall synthesizing enzymes. Future proteomic or ribosome profiling studies (Li et al., 2014) may be used to test these hypotheses.

\section{CONCLUDING REMARKS}

This manuscript deals with a fundamental question in bacteriology, relating cell dimensions and division to its nucleoid structure. The presumed signal(s) transmitted from the nucleoid (DNA) to its sacculus (PG) is crucial because both structures (macromolecules) are singular in a bacterium and essential for survival of the species. We suggest a function for the 
chromosome other than in replicating the genetic information and its flow from DNA to proteins.

The field of bacterial physiology, established in 1958, has since relied on genetics and chemistry. Involvement of physical forces at a higher (structural) level than sheer interactions between molecules, hardly dealt with in the literature, may open a new avenue to understand living matter. Participation of such forces in this signal needs pursuit. During the 20th century, physicists have been highly instrumental in advancing the quantitative biological sciences, e.g., the phage group led by Max Delbrück. Our call here is a far cry in the hope to devise methods that would measure the tiny intra-cellular forces, likely to take part in the functions discussed, cell division and shape determination.

Slowing replication rate by thymine limitation is a powerful tool to breach the minimal distance between successive replisomes with no detectable change in mass growth rate hence presumably cellular protein profile. In fast growing thymine-limited thy $A$ mutants, further divisions and replicationinitiations are delayed due to postponed replication-terminations and breaching the minimal distance-cumulatively so. The cascade of consequent reactions affect cell dimensions, overshooting length by default continuous elongasome activity initially, then width during the divisome activities until reaching a maximum that seems to be related to $N C$. The aberrant behavior of such cells can be exploited to decipher the mechanism(s) regulating the essential coupling between mass growth and the various biosynthetic activities of the nucleoid and the sacculus, and to discover the presumed primary signal relayed from DNA to the PG biosynthetic pathway in the divisome to simultaneously execute the division process and fix cell width. It is noteworthy that the postulated signal relayed from the nucleoid to the divisome may rather be related to the changes in gene concentration or relative gene dosage

\section{REFERENCES}

Amir, A. (2014). Cell size regulation in bacteria. Phys. Rev. Lett. 112:208102. doi: 10.1103/PhysRevLett.112.208102

Begg, K. J., and Donachie, W. D. (1978). Changes in cell size and shape in thyminerequiring Escherichia coli associated with growth in low concentrations of thymine. J. Bacteriol. 133, 452-458.

Bisson-Filho, A. W., Hsu, Y. P., Squyres, G. R., Kuru, E., Wu, F., Jukes, C., et al. (2017). Treadmilling by FtsZ filaments drives peptidoglycan synthesis and bacterial cell division. Science 355, 739-743. doi: 10.1126/science. aak9973

Campos, M., Govers, S. K., Irnov, I., Dobihal, G. S., Cornet, F., and Jacobs-Wagner, C. (2018). Genomewide phenotypic analysis of growth, cell morphogenesis, and cell cycle events in Escherichia coli. Molec. Syst. Biol. 14:e7573. doi: 10.15252/ msb. 20177573

Chandler, M. G., and Pritchard, R. H. (1975). The effect of gene concentration and relative gene dosage on gene output in Eschrichia coli. Molec. Gen. Genet. 138, 127-141. doi: 10.1007/bf02428117

Dion, M. F., Kapoor, M., Sun, Y., Wilson, S., Ryan, J., Vigouroux, A., et al. (2019). Bacillus subtilis cell diameter is determined by the opposing actions of two distinct cell wall synthetic systems. Nat. Microbiol. doi: 10.1038/s41564-0190439-02019. [Epub ahead of print].

Donachie, W. (1968). Relationships between cell size and time of initiation of DNA replication. Nature 219, 1077-1079. doi: 10.1038/2191077a0

Du, S., and Lutkenhaus, J. (2017). Assembly and activation of the Escherichia coli divisome. Molec. Microbiol. 105, 177-187. doi: 10.1111/mmi.13696 that reflect NC. Another alternative, testable theory (Gray et al., 2019), is that different nucleocytoplasmic ratios "can lead to different biophysical properties of the cytoplasm and hence to affect the mobility of large cytoplasmic objects." This theory and our $N C \rightarrow$ PG hypothesis need further and new experimental approaches to define the underlying physical and molecular mechanisms. We reckon that time will tell whether any of these hypotheses is closer to the "truth" (i.e., reality). The detailed, complicated picture summarized here is consistent with the existing observations, though meager at this stage of knowledge.

\section{AUTHOR CONTRIBUTIONS}

All authors listed have made a substantial, direct and intellectual contribution to the work, and approved it for publication.

\section{FUNDING}

This work was partially supported by a grant from the United States - Israel Binational Science Foundation (BSF \# 2017004, to AZ and JM) and Shenzhen Grants (JCYJ20170818164139781, KQTD2015033117210153, Engineering Laboratory [2016]1194).

\section{ACKNOWLEDGMENTS}

We gratefully acknowledge Conrad Woldringh for decades of cooperation and numerous discussions that crystallized the ideas and concepts inherent in this Perspective.

Egan, A. J., Cleverley, R. M., Peters, K., Lewis, R. J., and Vollmer, W. (2017). Regulation of bacterial cell wall growth. FEBS J. 284, 851-867. doi: 10.1111/ febs.13959

Egan, A. J. F., and Vollmer, W. (2013). “The physiology of bacterial cell division," in Annals of the New York Academy of Sciences 1277, issue "Antimicrobial Therapeutics Reviews", ed. K. Bush (New York, NY: The New York Academy of Sciences), 8-28. doi: 10.1111/j.1749-6632.2012.06818.x

Gray, W. T., Govers, S. K., Xiang, Y., Parry, B. R., Campos, M., Kim, S., et al. (2019). Nucleoid size scaling and intracellular organization of translation across bacteria. Cell 177, 1632-1648.

Helmstetter, H. E., Cooper, S., Pierucci, O., and Revelas, E. (1968). On the bacterial life sequence. Cold Spring Harbor Symp. Quant. Biol. 33, 809-822. doi: 10.1101/ SQB.1968.033.01.093

Höltje, J. V. (1998). Growth of the stress-bearing and shape-maintaining murein sacculus of Escherichia coli. Microbiol. Molec. Biol. Rev. 62, 181-203.

Jiménez-Sánchez, A. (2018). Equations of a Life. Algorithmic Analysis of the Bacterial cell Cycle and the Chromosome Replication. Germany: Lambert Academic Publishing.

Khan, S. R., Mahaseth, T. E., Kouzminova, A., Cronan, G. E., and Kuzminov, A. (2016). Static and dynamic factors limit chromosomal replication complexity in Escherichia coli, avoiding dangers of runaway overreplication. Genetics 202, 945-960. doi: 10.1534/genetics.115.184697

Kjeldgaard, N. O., Maaløe, O., and Schaechter, M. (1958). The transition between different physiological states during balanced growth of Salmonella typhimurium. J. Gen. Microbiol. 19, 607-616. doi: 10.1099/00221287-193-607 
Knox, S. S. (2018). Wave/particle duality and biomedical research designs. Molec. Biol. 7:215. doi: 10.4172/2168-9547.1000215

Knox, S. S., and Funk, R. H. W. (2014). Oncology and biophysics: a need for integration. J. Clin. Exp. Oncol. 2014, 1-6. doi: 10.4172/2324-9110.S1-001

Koch, A. L. (1993). Biomass growth rate during the prokaryote cell cycle. Crit. Rev. Microbiol. 19, 17-42. doi: 10.3109/10408419309113521

Kubitschek, H. E., Baldwin, W. W., Schroeter, S. J., and Graetzer, R. (1984). Independence of buoyant cell density and growth rate in Escherichia coli. J. Bacteriol. 158, 296-299.

Kurita, K., Shin, R., Tabei, T., and Shiomi, D. (2019). Relation between rotation of MreB actin and cell width of Escherichia coli. Genes Cells 24, 259-265. doi: $10.1111 /$ gtc. 12667

Li, G.-W., Burkhardt, D. J., Gross, C. C., and Weissman, J. S. (2014). Quantifying absolute protein synthesis rates reveals principles underlying allocation of cellular resources. Cell 157, 624-635. doi: 10.1016/j.cell.2014.02.033

Lin, J., and Amir, A. (2018). Homeostasis of protein and mRNA concentrations in growing cells. Nat. Commun. 9:4496. doi: 10.1038/s41467-018-06714-z

Maaløe, O., and Kjeldgaard, N. O. (1966). Control of Macromolecular Synthesis. San Francisco: WA Benjamin Inc.

Männik, J., and Bailey, M. W. (2015). Spatial coordination between chromosomes and cell division proteins in Escherichia coli. Front. Microbiol. 5:19. doi: 10.3389/ fmicb.2015.00306

Männik, J., Walker, B. E., and Männik, J. (2018). Cell cycle-dependent regulation of FtsZ in Escherichia coli in slow growth conditions. Molec. Microbiol. 110, 1030-1044. doi: $10.1111 / \mathrm{mmi} .14135$

Norris, V., den Blaauwen, T., Doi, R. H., Harshey, R. M., Janniere, L., JiménezSánchez, A., et al. (2007). Toward a hyperstructure taxonomy. Annu. Rev. Microbiol. 61, 309-329. doi: 10.1146/annurev.micro.61.081606.103348

O’Donnell, M., Langston, L., and Stillman, B. (2013). Principles and concepts of DNA replication in bacteria, archaea, and eukarya. Cold Spring Harbor Perspect. Biol. 5:a010108. doi: 10.1101/cshperspect.a010108

Ouzounov, N., Nguyen, J. P., Bratton, B. P., Jakobowitz, D., Gitai, Z., and Shaevitz, J. W. (2016). MreB orientation correlates with cell diameter in Escherichia coli. Biophys. J. 111, 1035-1043. doi: 10.1016/j.bpj.2016.07.017

Pritchard, R. H., Barth, P. T., and Collins, J. (1969). Control of DNA synthesis in bacteria. Microbial Growth Symp Soc. Gen. Microbiol. 19, 263-297.

Pritchard, R. H., and Zaritsky, A. (1970). Effect of thymine concentration on the replication velocity of DNA in a thymineless mutant of Escherichia coli. Nature 226, 126-131. doi: 10.1038/226126a0

Rabinovitch, A., Zaritsky, A., and Feingold, M. (2003). DNA-membrane interactions can localize bacterial cell. J. Theoret. Biol. 110, 1030-1044. doi: $10.1111 / \mathrm{mmi} .14135$

Schaechter, M., Maaløe, O., and Kjeldgaard, N. O. (1958). Dependency on medium and temperature of cell size and chemical composition during balanced growth of Salmonella typhimurium. J. Gen. Microbiol. 19, 592-606. doi: 10.1099/ 00221287-19-3-592

Shi, H., Bratton, B. P., Gitai, Z., and Huang, K. C. (2018). How to Build a Bacterial Cell: MreB as the Foreman of E. coli Construction. Cell 172, 1294-1305. doi: 10.1016/j.cell.2018.02.050

Stent, G. S. (1968). That was the molecular biology that was. Science 160, 390-395.

Stent, G. S., and Calendar, R. (1978). Molecular Genetics, 2nd Edn. San-Francisco: WH Freeman and Company.

Sueoka, N., and Yoshikawa, H. (1965). The chromosome of Bacillus subtilis. I. Theory of marker frequency analysis. Genetics 52, 747-757.

Taheri-Araghi, S., Bradde, S., Sauls, J. T., Hill, N. S., Levin, P. A., Paulsson, J., et al. (2014). Cell-Size control and homeostasis in bacteria. Curr. Biol. 25, 385-391. doi: 10.1016/j.cub.2014.12.009

Trueba, F. J., and Woldringh, C. L. (1980). Changes in cell diameter during the division cycle of Escherichia coli. J. Bacteriol. 142, 869-878.

Typas, A., Banzhaf, M., Gross, C. A., and Vollmer, W. (2012). From the regulation of peptidoglycan synthesis to bacterial growth and morphology. Nat. Rev. Microbiol. 10, 123-136. doi: 10.1038/nrmicro2677

Van der Ploeg, R., Verheul, J., Vischer, N. O., Alexeeva, S., Hoogendoorn, E., Postma, M., et al. (2003). Colocalization and interaction between elongasome and divisome during a preparative cell division phase in Escherichia col. Molec. Microbiol. 87, 1074-1087. doi: 10.1111/mmi.12150

van Teeffelen, S., Wang, S., Furchtgott, L., Huang, K. C., Wingreen, N. S., Shaevitz, J. W., et al. (2011). The bacterial actin MreB rotates, and rotation depends on cell-wall assembly. Proc. Natl. Acad. Sci. U. S. A. 108, 15822-15827. doi: 10.1073/pnas.1108999108

Woldringh, C. L. (2002). The role of co-transcriptional translation and protein translocation (transrtion) in bacterial chromosome segregation. Molec. Microbiol. 45, 17-29. doi: 10.1046/j.1365-2958.2002.02993.x

Woldringh, C. L., Grover, N. B., Rosenberger, R. F., and Zaritsky, A. (1980). Dimensional rearrangement of rod-shaped bacteria following nutritional shiftup. II. Experiments with Escherichia coli B/r. J. Theoret. Biol. 86, 441-454. doi: 10.1016/0022-5193(80)90344-6

Woldringh, C. L., Mulder, E., Valkenburg, J. A. C., Wientjes, F. B., Zaritsky, A., and Nanninga, N. (1990). Role of the nucleoid in the toporegulation of division. Res. Microbiol. 141, 39-49. doi: 10.1016/0923-2508(90) 90096-9

Yang, X. X., Lyu, Z. X., Miguel, A., McQuillen, R., Huang, K. C., and Xiao, J. (2017). GTPase activity-coupled treadmilling of the bacterial tubulin FtsZ organizes septal cell wall synthesis. Science 355:744. doi: 10.1126/science. aak9995

Zaritsky, A. (1975a). On dimensional determination of rod-shaped bacteria. J. Theoret. Biol. 54, 243-248. doi: 10.1016/s0022-5193(75)80129-9

Zaritsky, A. (1975b). Rate stimulation of DNA synthesis after inhibition. J. Bacteriol. 122, 841-846.

Zaritsky, A. (2015). Cell-shape homeostasis in Escherichia coli is driven by growth, division, and nucleoid complexity. Biophys. J. 109, 178-181. doi: 10.1016/j.bpj. 2015.06.026

Zaritsky, A., and Pritchard, R. H. (1971). Replication time of the chromosome in thymineless mutants of Escherichia coli. J. Molec. Biol. 60, 65-74. doi: 10.1016/ 0022-2836(71)90447-5

Zaritsky, A., and Pritchard, R. H. (1973). Changes in cell size and shape associated with changes in the replication time of the chromosome of Escherichia coli. J. Bacteriol. 114, 824-837.

Zaritsky, A., Rabinovitch, A., Liu, C., and Woldringh, C. L. (2017). Does the eclipse limit bacterial nucleoid complexity and cell width? Synth. Syst. Biotechnol. 2 , 267-275. doi: 10.1016/j.synbio.2017.11.004

Zaritsky, A., Van Geel, A., Fishov, I., Pas, E., Einav, M., and Woldringh, C. L. (1999). Visualizing multiple constrictions in spheroidal Escherichia coli cells. Biochimie 81, 897-900. doi: 10.1016/s0300-9084(00)87174-6

Zaritsky, A., Vischer, N., and Rabinovitch, A. (2007). Changes of initiation mass and cell dimensions by the "eclipse". Molec. Microbiol. 63, 15-21. doi: 10.1111/ j.1365-2958.2006.05501.x

Zaritsky, A., Wang, P., and Vischer, N. O. E. (2011). Instructive simulation of the bacterial cell division cycle. Microbiology 157, 1876-1885. doi: 10.1099/mic.0. 049403-0

Zaritsky, A., and Woldringh, C. L. (2015). Chromosome replication, cell growth, division and shape: a personal perspective. Front. Microbiol. 6:756. doi: 10.3389/ fmicb.2015.00756

Zaritsky, A., Woldringh, C. L., Einav, M., and Alexeeva, S. (2006). Thymine limitation and thymine starvation to study bacterial physiology and cytology. J. Bacteriol. 188, 1667-1679. doi: 10.1128/JB.188.5.1667-1679. 2006

Conflict of Interest Statement: The authors declare that the research was conducted in the absence of any commercial or financial relationships that could be construed as a potential conflict of interest.

Copyright () 2019 Zaritsky, Vollmer, Männik and Liu. This is an open-access article distributed under the terms of the Creative Commons Attribution License (CC BY). The use, distribution or reproduction in other forums is permitted, provided the original author(s) and the copyright owner(s) are credited and that the original publication in this journal is cited, in accordance with accepted academic practice. No use, distribution or reproduction is permitted which does not comply with these terms. 


\section{APPENDIX - GLOSSARY: PARAMETERS AND DEFINITIONS OF FIELD-SPECIFIC TERMS USED (THE CELL CYCLE AND DIMENSIONS)}

\section{Cell Growth and Cycle Parameters}

$\tau$, doubling time; $\tau_{m}$, of mass; $\tau_{d}$, between successive divisions

$C$, replication time, taken to duplicate the entire chromosome, from origin oriC to terminus $\operatorname{ter} C$

$D$, time between replication-termination and subsequent cell division

$\Lambda$, chromosome length in genome equivalents units

$E$, Eclipse - minimal possible distance $l_{\min }$ along the chromosome length $\Lambda$ needed for a replisome to be away from oriC before a succeeding replisome can initiate a next replication round there. In units of time, $E$ is proportional directly to the fraction $l_{\min } / \Lambda$ and to $C$, i.e., $E=C\left(l_{\min } / \Lambda\right)$

$M i$, initiation mass - cell mass per number of oriC at the time of replication-initiation

$n$, number of replisome positions, equal to $C / \tau$

$N C$, nucleoid complexity - amount of DNA in genome equivalents associated with a single terC, equals to $\left(2^{n}-1\right) /(n \times \ln 2)$

$x$, distance of a gene from oriC in units of $\Lambda$

\section{Cell Dimensions and Composition}

$M$, average cell mass in a steady-state culture growing in batch, equal to $\ln 2 \times M i \times 2^{(C+D) / \tau}$

$L$, cell length

$V$, cell volume

$W$, cell width

$G$, amount of DNA per cell in genome equivalents

$P G$, peptidoglycan

[T], concentration of thymine supplied to growth media of thyA mutants

\section{Hyperstructures and Processes}

Divisome: A contractile ring of polypeptides involved in bacterial cell division

Replisome: A matrix of enzymes that is the site of DNA replication

Transertion: Co-transcriptional translation of membrane protein genes coupled to insertion of these proteins to the membrane 